\title{
A INFLUÊNCIA DA VARIAÇÃO SAZONAL DAS CHUVAS NO VOLUME DE CISTERNAS DE ÁGUA NO ESTADO DA BAHIA
}

\author{
Juliana Farias Araujoㅜ; Eduardo Cohim² \\ 1. Bolsista PIBIC/CNPq, Graduando em Engenharia Civil, Universidade Estadual de Feira de Santana, e-mail: \\ julianafariaz1996@gmail.com \\ 2. Eduardo Cohim, Departamento de Tecnologia, Universidade Estadual de Feira de Santana, e-mail: edcohim@gmail.com
}

PALAVRAS-CHAVE: Cisternas, chuvas, Bahia.

\section{INTRODUÇÃO}

O programa de Formação e Mobilização Social para a Convivência com o Semiárido P1MC - baseia-se na construção de cisternas com volume definido em 16.000 L. Porém, questionou-se essa padronização, a fim de que se maximize a relação custo-benefício do Programa e garanta o real atendimento das necessidades hídricas de cada habitação. Assim, buscou-se desenvolver métodos de dimensionamento de reservatório mais racionais, de forma que se leve em consideração a diversidade de fatores que envolvem a determinação da confiabilidade, como, principalmente, a pluviosidade (média pluviométrica e a sua distribuição anualmente), alé, da área de telhado e a demanda de cada habitação. Para analisar as diversas combinações dessas variáveis, buscou-se utilizar parâmetros adimensionais e normalizados, como a Fração de Demanda $\left(\mathrm{F}_{\mathrm{D}}\right)$ e a Fração de Reservação $\left(\mathrm{F}_{\mathrm{R}}\right)$. Neste estudo, utilizou-se a confiabilidade de $95 \%$, por se direcionar para áreas rurais, em que a água de chuva é a principal fonte hídrica. Assim, buscou-se o volume do reservatório recomendável para cada residência instalada no território baiano, de forma que se garanta que não haja um superdimensionamento, nem um subdimensionamento. Houve também uma indicação da área de telhado adicional, quando necessária.

\section{MATERIAL E MÉTODOS OU METODOLOGIA (ou equivalente)}

Inicialmente, comprovou-se que, juntamente com o total pluviométrico, a análise da distribuição das chuvas apresenta extrema relevância. Por exemplo, um ano em que os totais pluviométricos tenham sido em torno da média pluviométrica pode, mesmo assim, apresentar restrições, se ocorrer período de estiagem. (NOBRE E MELO, 2001).

Foram empregados dados de chuvas diárias de 180 postos distribuídos por todo o território ,com séries recentes e extensão de 15 anos (92 postos) e 30 anos (88 postos). Os dados utilizados nesse estudo foram disponíveis no HidroWeb da Agência Nacional de Águas (ANA) e no Banco de Dados de Recursos Hídricos (BDRH) do Instituto do Meio Ambiente e Recursos Hídricos da Bahia (INEMA).

Neste estudo, adotou-se o valor e confiabilidade igual a $95 \%$ tendo em vista o direcionamento para áreas rurais, onde a água de chuva é a principal fonte de água para uso humano.

Para expressar a diversidade de combinação de valores das variáveis envolvidas, utilizaram-se parâmetros normalizados, como a Fração de Demanda (FD) e a Fração de Reservação (FR) (FEWKES, 1999). Esses parâmetros já foram utilizados por Campisano e Modica (2012) quando determinaram o volume ótimo baseado no critério do mínimo custo em na Sicília na Itália. 
Para realizar a determinação do volume de reservatório, desenvolveu-se uma rotina na plataforma Matlab, em que se busca a FD que pode ser atendida com uma dada confiabilidade, para os diversos volumes de reservatórios, nos diversos postos pluviométricos baianos. Solicitam-se dados de entradas, como dados de precipitação do posto pluviométrico correspondente ao município desejado. Para a simulação, usa-se o modelo comportamental que simula a operação de um reservatório em um período de tempo por meio de algoritmos de fluxos de massas.

Utilizou-se um algoritmo mais generalizado para a operação de um reservatório (FEWKES, 1999). Mashford e Maheepala (2014) afirmam que esse modelo mostrou ser uma aproximação na melhor das hipóteses para se calcular o rendimento exato durante qualquer período e o volume a qualquer momento durante a operação do tanque.

Em seguida, foi definida uma Fração de Demanda Recomendável por perceber que há um acréscimo cada vez menor do atendimento à demanda em razão de um incremento do volume das cisternas. Para encontrar esse valor, fez-se o uso neste trabalho das ferramentas matemáticas, em que se busca aquele para qual a derivada da curva FD x FR vale 1. Escolheuse esse valor por indicar uma variação na Fração de Demanda corresponde a uma mesma variação na Fração de Reservação. Assim, o aumento de $1 \mathrm{~m}^{2}$ do volume da cisterna, resulta em um ganho de $1 \mathrm{~m}^{3}$ para o atendimento da demanda hídrica da família.

Buscou também um ajuste de uma curva exponencial que descreve o comportamento aproximado da Fração de Reservação em relação à Fração de Demanda para cada um dos postos pluviométrico. Para comprovar a adequabilidade do uso dessa curva, houve a análise do resíduo entre do FR calculado exponencialmente e o FR encontrado por meio do fluxo de massa.

Para definir essas curvas, foi necessário calcular dois coeficientes da curva: $\mathrm{K}$ e $\alpha$, a partir do estudo de regressão dos pares de FD e FR encontrados por meio do fluxo de massa, em que os valores de FD variou de 0 até o ponto máximo, ou seja, ponto em que a confiabilidade se torna independente do aumento no tamanho do tanque (Imteaz et al., 2012).

Para facilitar o uso da ferramenta, foram construídas quatro curvas de isoquantas, por meio do Software Surfer. Essas curvas estão distribuídas no mapa do estado da Bahia e possui valores da precipitação da localidade, da Fração de Demanda Recomendável e dos valores dos coeficientes da curva exponencial. Além disso, possibilita-se obter esses valores aproximados para locais em que não se realizou a análise, por meio da interpolação realizada para a construção das isoquantas.

Percebeu-se que, em casos que a Fração de Demanda da residência supera a Fração de Demanda Recomendável, é oportuna uma ampliação inicial na área de coleta do que o aumento do volume, a fim de que seja atendida a demanda dos moradores das casas com uma dada confiabilidade estabelecida.

\section{RESULTADOS E/OU DISCUSSÃO (ou Análise e discussão dos resultados)}

Após determinar os coeficientes da curva exponencial para cada posto pluviométrico, foram construídos quatro mapas. O primeiro contém a isoquanta do coeficiente $\mathrm{K}$, enquanto que o segundo contém a isoquanta do coeficiente $\alpha$. Esses mapas estão exibidos a seguir, na Figura 1 e na Figura 2, respectivamente. Além disso, houve a construção do mapa que contém a precipitação média dos postos localizados no estado da Bahia (Figura 3), assim como o que possui os valores da FD recomendável (Figura 4). 


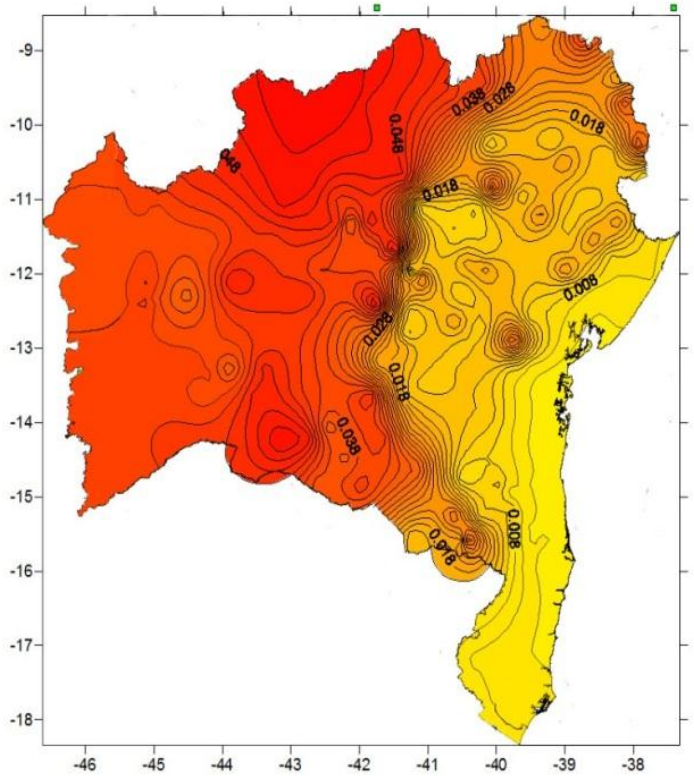

Figura 1- Apresenta as isoquantas do parâmetro $\propto$

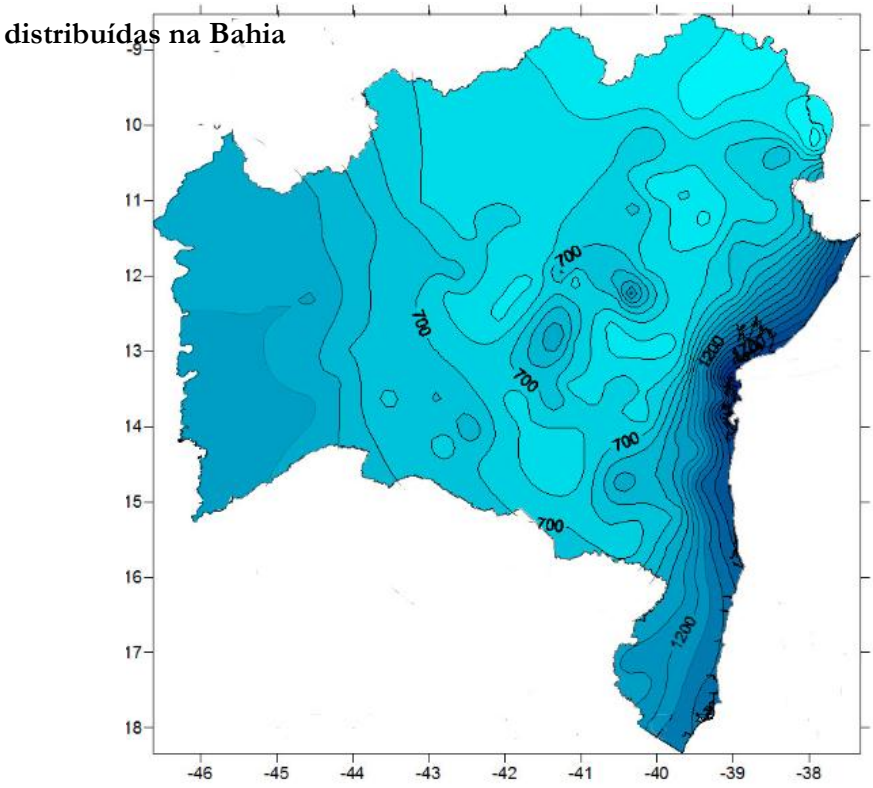

Figura 3- Apresenta as isoquantas da média de precipitação distribuída na Bahia

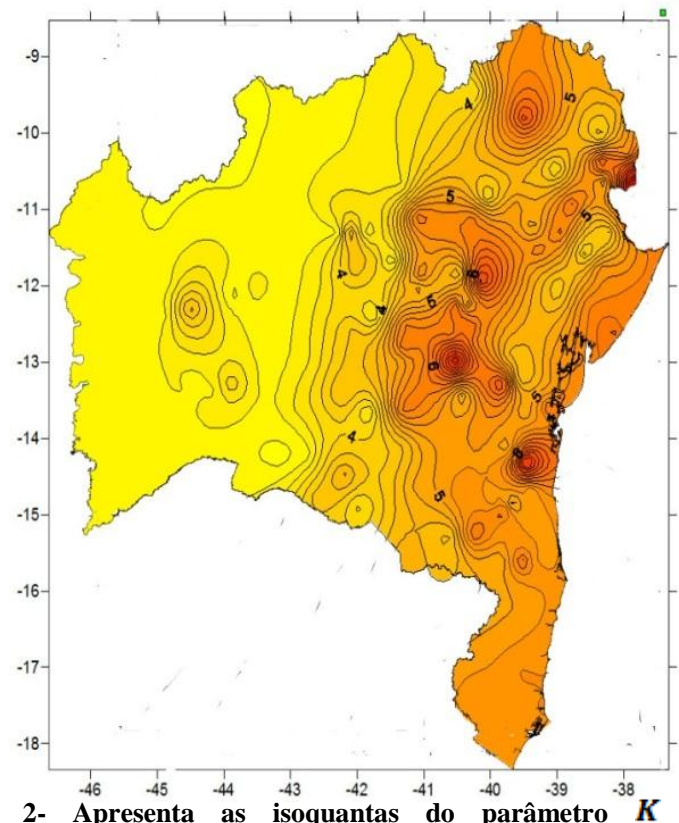

Figura 2- Apresenta as isoquantas do parâmetro ${ }^{-38} K$

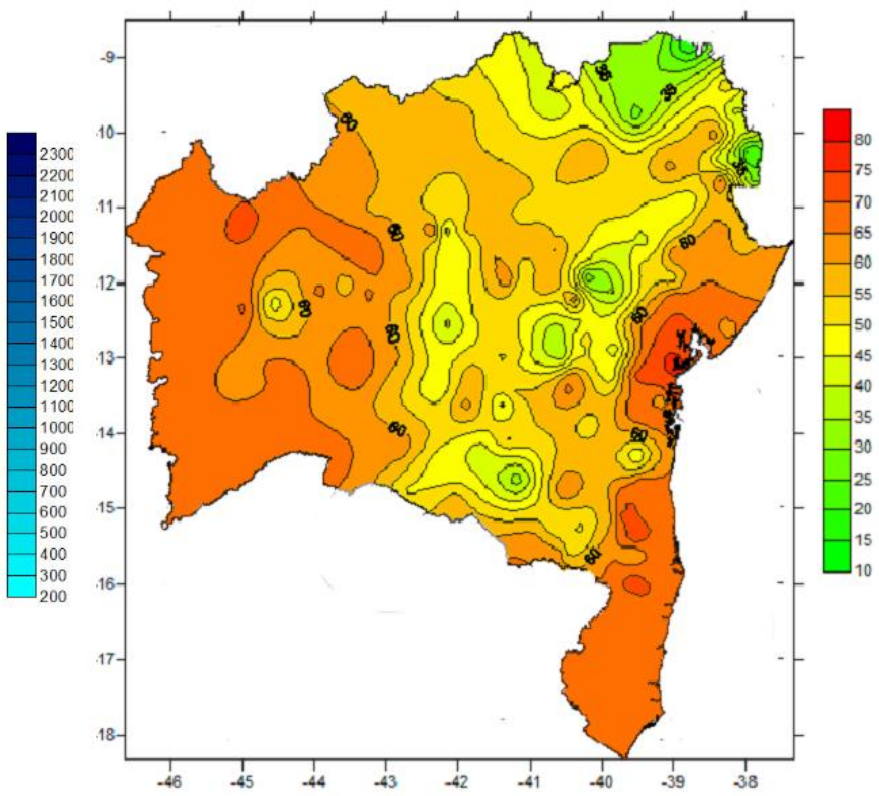

Figura 4- Apresenta as isoquantas do $F_{D}$ recomendável distribuídas na Bahia

Esses mapas serão fundamentais para se obter o volume do reservatório para todo o território baiano, inclusive para locais em que não se dispõem de dados pluviométricos.

\section{CONSIDERAÇÕES FINAIS (ou Conclusão)}

Neste trabalho, percebe-se que há uma grande influência da sazonalidade das chuvas no dimensionamento do reservatório, principalmente no estado da Bahia, por apresentar um cenário com alta variabilidade na precipitação pluvial. Isso decorre da união de diferentes sistemas meteorológicos que atuam nessa região, tais como, Zona de Convergência Intertropical (ZCIT), Vórtices Ciclônicos (VCAN), Sistemas Frontais (FPA), Zona de Convergência do Atlântico Sul (ZCAS), ondas de leste, brisas marítimas/terrestres e ventos vale/montanha, bem como de um relevo constituído por planícies, vales, serras e montanhas, (BARSOSA, 2000; BRAGA, et al. 1998). 
Por conta disso, concluiu-se que há a falta de adequabilidade do padrão de 16.000L ( volume padrão definido pelo Programa 1 Milhão de Cisternas ) para a construção de reservatórios de água pluvial para todos os estado da Bahia.

Para melhorar o dimensionamento dos reservatórios, houve a necessidade do desenvolvimento de uma ferramenta capaz de analisar as variáveis referentes ao dimensionamento do reservatório, como a pluviosidade, demanda, área de telhado e confiabilidade. Para isso, utilizou-se de parâmetros como a Fração de Demanda e Fração de Reservação, de mapas de isoquantas de parâmetros que definem as curvas de FR x FD e de dados de pluviosidade de 15 ou 30 anos de 180 postos de pluviosidade baianos.

Ao longo do desenvolvimento da pesquisa, ratificou-se que, juntamente com o total pluviométrico, a análise da distribuição das chuvas apresenta extrema relevância. Por exemplo, um ano em que os totais pluviométricos tenham sido em torno da média pluviométrica pode, mesmo assim, apresentar restrições, se ocorrer período de estiagem. Dessa forma, há uma crítica contra muitos métodos já existentes, inclusive os presentes na NBR 15527:2007, em que se utiliza apenas a média pluviométrica para fazer o dimensionamento do reservatório.

Dessa forma, percebe-se que este trabalho representa uma boa contribuição, pois oferece uma ferramenta prática e confiável para que se determine o tamanho ótimo das cisternas que atendam as reais necessidades hídricas de cada habitação, sem que necessite fazer um trabalho fastidioso para se determinar o volume. É solicitado apenas as coordenadas geográficas da habitação no mapa da Bahia (pode ser obtida com o uso de um GPS), o número de moradores, e a área de telhado da casa.

\section{REFERÊNCIAS}

ANA - AGÊNCIA NACIONAL DE ÁGUA. HIDROWEB. Disponível em: < http://hidroweb.ana.gov.br/>. Acesso em: 05.set.2015.

ASA PROGRAMA 1 MILHÃO DE CISTERNAS: Resultados. Disponível em: http://www.asabrasil.org.br/Portal/Informacoes.asp?COD_MENU=1558\&WORDKEY=. Resultados. Acesso em 07.jun.2016.

ASSOCIAÇÃO BRASILEIRA DE NORMAS TÉCNICAS. NBR 15527: água de chuva: aproveitamento em áreas urbanas para fins não potáveis: requisitos. Rio de Janeiro, 2007.

BRAGA, C. C.; MELO, M. L. D.; MELO, E. C. S. Análise de agrupamento aplicada a distribuição da precipitação no Estado da Bahia. In: Congresso Brasileiro de Meteorologia, 10, 1998, Brasília. Anais... Brasília: Sociedade Brasileira de Meteorologia, 1998. CD-Room.

CAMPISANO A.; MODICA C. Optimal sizing of storage tanks for domestic rainwater harvesting in Sicily Resources. Conservation and Recycling, 63, pp. 9-16, 2012.

FEWKES, A. Modelling the performance of rainwater collection systems: towards a generalized approach. Urban Water v.1, n. 4, p. 323-333, 1999.

GOMES, J.; WEBER, D. C.; DELONG, C. M. Dimensionamento de Reservatórios de Armazenamento de Águas Pluviais, usando um Critério Financeiro. Revista Brasileira de Recursos Hídricos, v.15, n.1, p.89-100, mar. 2010.

IMTEAZ, M.A.; RAHMAN, A.; AHSAN, A. Reliability analysis of rainwater tanks: A comparison between south-east and central Melbourne. Resour. Conserv. Recycl., 66(1), 1-7, 2012.

MASHFORD, John; MAHEEPALA, Shiroma. A general model for the exact computation of yield from a rainwater tank. Applied Mathematical Modelling, 39, 2015.

NOBRE, P.; MELO, A. B. C. Variabilidade climática intrasazonal sobre o Nordeste do Brasil em 1998-2000. Climanálise, Brasil, 2001. 